\section{OPEN ACCESS}

Edited by:

Jesus Martin-Vaquero,

University of Salamanca, Spain

Reviewed by:

Praveen Agarwal,

Anand International College of

Engineering, India

Ding-fang Zeng,

Beijing University of Technology, China

*Correspondence: Abdelhalim Ebaid aebaid@ut.edu.sa

Specialty section

This article was submitted to Mathematical Physics,

a section of the journal

Frontiers in Physics

Received: 02 November 2019

Accepted: 13 February 2020

Published: 06 March 2020

Citation:

Alanazi AM, Ebaid A, Alhawiti WM and

Muhiuddin G (2020) The Falling Body

Problem in Quantum Calculus.

Front. Phys. 8:43

doi: 10.3389/fphy.2020.00043

\title{
The Falling Body Problem in Quantum Calculus
}

\author{
Abdulaziz M. Alanazi, Abdelhalim Ebaid*, Wadha M. Alhawiti and Ghulam Muhiuddin \\ Department of Mathematics, Faculty of Sciences, University of Tabuk, Tabuk, Saudi Arabia
}

The quantum calculus, $q$-calculus, is a relatively new branch in which the derivative of a real function can be calculated without limits. In this paper, the falling body problem in a resisting medium is revisited in view of the $q$-calculus to the first time. The $q$-differential equations describing the vertical velocity and distance of the body are obtained. Accordingly, exact expressions for the vertical velocity and the vertical distance are provided. The solutions are expressed in terms of the small $q$-exponential function which is an elementary function in the $q$-calculus. The dimensionality of the obtained formulae of the velocity and the distance are also analyzed. In addition, the present exact solutions reduce to the corresponding solutions in classical Newtonian mechanics when the quantum parameter $q$ tends to one.

Keywords: $q$-calculus, $\boldsymbol{q}$-series, exact solution, falling body problem, $\boldsymbol{q}$-differential equation

\section{Mathematics Subject Classification:35A24, 49K15}

\section{INTRODUCTION}

Basically, the regular calculus uses limits in calculating the derivatives of real functions. However, the calculus without limits is nowadays known as quantum calculus or $q$-calculus. Historically, in the eighteenth century, Euler obtained the basic formulae in $q$-calculus. However, Jackson [1] may have been the first to introduce the notion of the definite $q$-derivative and $q$-integral. Currently, there is a significant interest in implementing the $q$-calculus due to its applications in several areas, such as mathematics, number theory, and combinatorics [2]. Ernst [3,4] pointed out that the majority of scientists who use $q$-calculus are physicists. Baxter [5] introduced the exact solutions of several models in Statistical Mechanics. Bettaibi and Mezlini [6] solved some $q$-heat and $q$-wave equations. Many interesting results in such area of research were also introduced by several authors in the literature [7-12].

In this paper, we aim to extend the applications of the $q$-calculus to study the falling body problem in a resisting medium. This problem and also the full projectile motion have been investigated by several authors [13-17] using various definitions in fractional calculus. However, the present paper may be the first to analyze the falling body problem in view of the $q$-calculus.

The basic formulae in $q$-calculus will be used to analyze the motion of a falling body in a resisting medium. Moreover, it will be shown that the exact solutions for the vertical velocity and distance reduce to the classical ones as $q \rightarrow 1$. The paper is organized as follows. Section 2 presents the main aspects of the $q$-calculus. Sections 3 discusses the application of the $q$-calculus on the falling body problem. Section 4 includes an additional analysis. Finally, section 5 outlines the conclusions. 


\section{THE MAIN ASPECTS OF THE $q$-CALCULUS}

Let $q \in \mathbb{R}$ and $n \in \mathbb{N}$, then $[n]_{q}$ is defined as (first chapter in [18])

$$
[n]_{q}=\frac{1-q^{n}}{1-q}
$$

and as $q \rightarrow 1$, we have

$$
\lim _{q \rightarrow 1}[n]_{q}=n .
$$

The $q$-factorial $[n]_{q}$ ! of a positive integer $n$ is given by

$$
[n]_{q} !=[1]_{q} \times[2]_{q} \times[3]_{q} \times \cdots \times[n]_{q} .
$$

The definition of $q$-differential is $d_{q} f(t)=f(t)-f(q t)$ and the $q$-derivative of a function $f(t)$ is defined by [18]

$$
D_{q} f(t):=\frac{d_{q} f(t)}{d_{q} t}=\frac{f(t)-f(q t)}{(1-q) t}, \quad t \neq 0,
$$

such that

$$
\lim _{q \rightarrow 1} D_{q} f(t)=f^{\prime}(t)
$$

if $f$ is differentiable at $t$, and we have at $t=0$ that

$$
D_{q} f(0)=\lim _{t \rightarrow 0} D_{q} f(t) .
$$

According to (4) we have

$$
D_{q} t^{n}=[n]_{q} t^{n-1} .
$$

The small $q$-analog of the exponential function $e^{t}$ denoted by $e_{q}(t)$ (also called the small $q$-exponential function) is given as

$$
e_{q}(t)=\sum_{j=0}^{\infty} \frac{t^{j}}{[j]_{q} !} \text {. }
$$

The definite Jackson $q$-integral is defined by

$$
\int_{0}^{x} f(t) d_{q} t=(1-q) x \sum_{j=0}^{\infty} q^{j} f\left(q^{j} x\right),
$$

and according to (4) and (9), we have

$$
\int_{0}^{x} D_{q} f(t) d_{q} t=f(x)-f(0) .
$$

The indefinite Jackson $q$-integral of the small $q$-exponential function $e_{q}(\alpha t)$ is given as [18]

$$
\int e_{q}(\alpha t) d_{q} t=\frac{1}{\alpha} e_{q}(\alpha t)+c
$$

where $c$ is a real constant. The correctness of dimensionality of the physical quantities is actually guaranteed by the definition (4).

\section{THE FALLING BODY PROBLEM}

Consider the falling of an object of mass $m$ in the Earth gravitational field through the air from a height $h$ with initial velocity $v_{0}$. The classical equation of motion for the particle is given by $[15,16]$

$$
m \frac{d v}{d t}=-m g-m k v
$$

where $k$ is a positive constant and its dimensionality is the inverse of seconds, i.e., $[k]=s^{-1}$. The initial conditions are given as

$$
v(0)=v_{0}, \quad z(0)=h,
$$

where $z(t)$ is the vertical distance of the particle at arbitrary time $t$ and $\frac{d z(t)}{d t}=v(t)$. The equation of motion (12) in view of the quantum calculus becomes

$$
\frac{d_{q} v}{d_{q} t}:=-g-k v, \quad q \in(0,1] .
$$

In order to solve Equation (14), we assume the solution in the series form:

$$
v(t)=\sum_{n=0}^{\infty} a_{n} t^{n}
$$

and therefore

$$
\begin{aligned}
\frac{d_{q} v}{d_{q} t} & =\sum_{n=0}^{\infty}[n]_{q} a_{n} t^{n-1} \\
& =\sum_{n=1}^{\infty}[n]_{q} a_{n} t^{n-1}, \quad \text { where } \quad[0]_{q}=0 \\
& =\sum_{n=0}^{\infty}[n+1]_{q} a_{n+1} t^{n}
\end{aligned}
$$

Substituting (15) and (16) into (14), yields

$$
\sum_{n=0}^{\infty}[n+1]_{q} a_{n+1} t^{n}=-g-k \sum_{n=0}^{\infty} a_{n} t^{n}
$$

or

$$
[1]_{q} a_{1}+\sum_{n=1}^{\infty}[n+1]_{q} a_{n+1} t^{n}=-g-k a_{0}-k \sum_{n=1}^{\infty} a_{n} t^{n}
$$

which gives

$$
\begin{aligned}
a_{1} & =\frac{-g-k a_{0}}{[1]_{q}}, \\
a_{n+1} & =\frac{-k a_{n}}{[n+1]_{q}}, \quad n \geq 1,
\end{aligned}
$$


From (19), we have

$$
\begin{aligned}
& a_{2}=\frac{-k a_{1}}{[2]_{q}}=\frac{(-1)^{2} k g+(-k)^{2} a_{0}}{[1]_{q}[2]_{q}}, \\
& a_{3}=\frac{-k a_{2}}{[3]_{q}}=\frac{(-1)^{3} k^{2} g+(-k)^{3} a_{0}}{[1]_{q}[2]_{q}[3]_{q}}, \\
& a_{4}=\frac{-k a_{3}}{[4]_{q}}=\frac{(-1)^{4} k^{3} g+(-k)^{4} a_{0}}{[1]_{q}[2]_{q}[3]_{q}[4]_{q}}, \\
& \cdot \quad n \geq 1 . \\
& a_{n}=\frac{(-1)^{n} k^{n-1} g+(-k)^{n} a_{0}}{[1]_{q}[2]_{q}[3]_{q} \ldots[n]_{q}}, \quad n \geq
\end{aligned}
$$

This $n$-term coefficient can expressed in terms of the $q$-factorial $[n]_{q}$ ! as

$$
a_{n}=\frac{(-1)^{n} k^{n-1} g+(-k)^{n} a_{0}}{[n]_{q} !}, \quad n \geq 1
$$

The instantaneous velocity is obtained as

$$
\begin{aligned}
v(t) & =a_{0}+\sum_{n=1}^{\infty} a_{n} t^{n}, \\
& =a_{0}+\sum_{n=1}^{\infty}\left[\frac{(-1)^{n} k^{n-1} g+(-k)^{n} a_{0}}{[n]_{q} !}\right] t^{n} . \\
& =a_{0}+\sum_{n=1}^{\infty}\left[\frac{(g / k)(-k t)^{n}+(-k t)^{n} a_{0}}{[n]_{q} !}\right],
\end{aligned}
$$

which can be written as

$$
v(t)=a_{0}+\left(\frac{g}{k}+a_{0}\right) \sum_{n=1}^{\infty} \frac{(-k t)^{n}}{[n]_{q} !} .
$$

In terms of the small exponential function $e_{q}(-k t)$, we have

$$
v(t)=a_{0}+\left(\frac{g}{k}+a_{0}\right)\left[e_{q}(-k t)-1\right] .
$$

Applying the first initial condition in (13) on (24), we obtain $a_{0}=v_{0}$ and therefore $v(t)$ becomes

$$
v(t)=v_{0}+\left(\frac{g}{k}+v_{0}\right)\left[e_{q}(-k t)-1\right]
$$

which can be simplified as

$$
v(t)=-\frac{g}{k}+\left(\frac{g}{k}+v_{0}\right) e_{q}(-k t)
$$

The vertical distance $z(t)$ in quantum calculus is governed by,

$$
D_{q} z(t)=-\frac{g}{k}+\left(\frac{g}{k}+v_{0}\right) e_{q}(-k t)
$$

where $v(t)=D_{q} z(t)$. Integrating (27), it then follows;

$$
\int_{0}^{t} D_{q} z(\tau) d_{q} \tau=\int_{0}^{t}\left(-\frac{g}{k}\right) d_{q} \tau+\left(\frac{g}{k}+v_{0}\right) \int_{0}^{t} e_{q}(-k \tau) d_{q} \tau,
$$

and hence,

$$
z(t)-z(0)=-\frac{g}{k}\left[\frac{\tau}{[1]_{q}}\right]_{0}^{t}+\left(\frac{g}{k}+v_{0}\right)\left[-\frac{e_{q}(-k \tau)}{k}\right]_{0}^{t},
$$

or

$$
z(t)=h-\frac{g}{k}\left(\frac{t}{[1]_{q}}\right)+\left(\frac{g}{k}+v_{0}\right)\left(-\frac{e_{q}(-k t)}{k}+\frac{1}{k}\right),
$$

i.e.,

$$
z(t)=h-\frac{g t}{k}+\frac{1}{k}\left(\frac{g}{k}+v_{0}\right)\left(1-e_{q}(-k t)\right),
$$

where $[1]_{q}=1$. The exact solutions (26) and (31) should be reduced to the corresponding solutions in classical Newtonian mechanics when $q \rightarrow 1$. In addition, if the acceleration due to gravity is measured in $m s^{-2}$, then the vertical velocity in (26) must has dimension $m s^{-1}$ and the vertical distance in (31) must has dimension $m$. These issues are addressed in the following section.

\section{ANALYSIS AND APPLICATIONS}

First of all, we investigate the solutions (26) and (31) when $q \rightarrow 1$. In this case, the small exponential function $e_{q}(-k t)$ reduces to the standard exponential function $e^{-k t}$ in classical calculus. Hence, (26) becomes

$$
v(t)=-\frac{g}{k}+\left(v_{0}+\frac{g}{k}\right) e^{-k t},
$$

which is the analytic expression for velocity in the case of the classical Newtonian mechanics (see Equation 16 in reference [15]). Besides, the vertical distance in (31) reduces to

$$
z(t)=h-\frac{g t}{k}+\frac{1}{k}\left(\frac{g}{k}+v_{0}\right)\left(1-e^{-k t}\right),
$$

which is also the analytic expression for the vertical distance in the classical Newtonian mechanics (see Equation 17 in reference [15]).

In addition, in the case of no air resistance, i.e., the parameter $k$ vanishes, we obtain from (32) that

$$
\begin{aligned}
\left.v(t)\right|_{k \rightarrow 0} & =\lim _{k \rightarrow 0}\left[v_{0} e^{-k t}+g\left(\frac{e^{-k t}-1}{k}\right)\right], \\
& =v_{0}+g \lim _{k \rightarrow 0}\left(\frac{e^{-k t}-1}{k}\right), \\
& =v_{0}+g \lim _{k \rightarrow 0}\left(\frac{-t e^{-k t}}{1}\right), \\
& =v_{0}-g t .
\end{aligned}
$$


Also, the vertical distance in (33) in the absence of air resistance becomes

$$
\begin{aligned}
\left.z(t)\right|_{k \rightarrow 0} & =h+\lim _{k \rightarrow 0}\left[\frac{-g t k+\left(g+k v_{0}\right)\left(1-e^{-k t}\right)}{k^{2}}\right], \\
& =h+\lim _{k \rightarrow 0}\left[\frac{v_{0}-g t+\left[\left(g+k v_{0}\right) t-v_{0}\right] e^{-k t}}{2 k}\right], \\
& =h+\lim _{k \rightarrow 0}\left[\frac{-\left[\left(g+k v_{0}\right) t-v_{0}\right] t e^{-k t}+v_{0} t e^{-k t}}{2}\right] \\
& =h+\left(\frac{-\left(g t-v_{0}\right) t+v_{0} t}{2}\right) \\
& =h+v_{0} t-\frac{1}{2} g t^{2} .
\end{aligned}
$$

Here, it should be noted that L'Hôpital's rule was applied to calculate the above limits. The Equations (34) and (35) are the same of the corresponding equations for the vertical velocity and vertical distance in Newtonian mechanics in the absence of air resistance.

Regarding the dimensions of the $q$-forms of $v(t)$ and $z(t)$ in (26) and (31), respectively, it should be first to specify the dimensions of the quantities $e_{q}(-k t)$ and $\left(1-e_{q}(-k t)\right)$ as indicated below:

$$
\begin{aligned}
& {[k t]=[k] \times[s]=s^{-1} \times s=\text { Scalar, }} \\
& {\left[e_{q}(-k t)\right]=\text { Scalar, }} \\
& {\left[1-e_{q}(-k t)\right]=\text { Scalar. }}
\end{aligned}
$$

By this, $e_{q}(-k t)$ and $\left(1-e_{q}(-k t)\right)$ are dimensionless quantities, i.e., $e_{q}(-k t)$ and $\left(1-e_{q}(-k t)\right)$ are scalar quantities. Accordingly, $v(t)$ in (26) always has dimension $m s^{-1}$ for all values of the quantum parameter $q$. Also $z(t)$ in (31) always has dimension $m \forall q \in(0,1]$. The correctness of dimensions of the $q$ vertical velocity and the $q$-height was actually guaranteed by the definition (4) without any need to involve an auxiliary parameter as in the literature $[15,16]$.

Although the present model of the falling body problem seems simple, the authors believe that the current work is worthy of

\section{REFERENCES}

1. Jackson FH. On a q-definite integrals. Q J Pure Appl Math. (1910) 41:193-203.

2. Andrews GE. q-Series: Their Development and Applications in Analysis, Number Theory, Combinatorics, Physics and Computer Algebra. CBMS Regional Conference Series in Mathematics, Vol. 66. Providence, RI: American Mathematical Society (1986).

3. Ernst T. The history of q-calculus and a new method (Licentiate thesis), U.U.D.M. Report 2000: 16. Available online at: https://pdfs.semanticscholar. org/895b/4f8b059dc7ad18807f5aabdf09f2158d0c66.pdf

4. Ernst T. A method for $q$-calculus. J Nonlin Math Phys A Method. (2003) 10:487-525. doi: 10.2991/jnmp.2003.10.4.5

5. Baxter R. Exact Solved Models in Statistical Mechanics. New York, NY: Academic Press (1982).

6. Bettaibi N, Mezlini K. On the use Of the $q$-Mellin transform to solve some $q$-heat and $q$-wave equations. Int J Math Arch. (2012) 3:446-55. exploration. This is because the present solution was provided to the first time for the falling problem in view of $q$-calculus. In addition, it was shown in this paper the way of obtaining the solutions in exact forms and also how to check the dimensions of the physical quantities in terms of $q$-parameter. Furthermore, the obtained solutions can be verified by direct substitutions into the governing equations. Therefore, the present work is a first step for further studies in future to explore various physical models in applied mathematics implementing the $q$-calculus.

\section{CONCLUSION}

In this paper, the quantum calculus was applied to solve the falling body problem. The exact solutions for the $q$ vertical velocity and the $q$-distance have been obtained. The obtained exact solutions were expressed in terms of the small $q$-exponential function. The correctness of dimensionality of the obtained formulae of the velocity and the distance was proved. Moreover, The present exact solutions reduced to the corresponding solutions in classical Newtonian mechanics when the quantum parameter $q$ tends to one. The present work can be further extended to explore the physical properties of the projectile motion in two and three dimensions in view of the $q$-calculus.

\section{DATA AVAILABILITY STATEMENT}

All datasets generated for this study are included in the article/supplementary material.

\section{AUTHOR CONTRIBUTIONS}

All authors listed have made a substantial, direct and intellectual contribution to the work, and approved it for publication.

\section{FUNDING}

The authors extend their appreciation to the Deanship of Scientific Research at University of Tabuk for funding this work through Research Group no. RGP-0207-1440.

7. Li YQ, Sheng ZM. A deformation of quantum mechanics. J Phys A Math Gen. (1992) 25:6779-88. doi: 10.1088/0305-4470/25/24/028

8. Annaby MH, Mansour ZS. q-Fractional Calculus and Equations. Heidelberg; New York, NY: Springer (2012).

9. Koca I, Demirci E. On local asymptotic stability of $q$-fractional nonlinear dynamical systems. Appl Appl Math. (2016) 11:174-83. Available online at: http://pvamu.edu/aam

10. Rangaig NA, Pada CT, Convicto VC. On the existence of the solution for $q$ Caputo fractional boundary value problem. Appl Math Phys. (2017) 5:99-102. doi: 10.12691/amp-5-3-4

11. Tang Y, Zhang T. A remark on the $q$-fractional order differential equations. Appl Math Comput. (2019) 350:198-208. doi: 10.1016/j.amc.2019. 01.008

12. Chanchlani L, Alha S, Gupta J. Generalization of Taylor's formula and differential transform method for composite fractional $q$-derivative. Ramanujan J. (2019) 48:21-32. doi: 10.1007/s11139-018-9997-7 
13. Kwok SF. A falling body problem through the air in view of the fractional derivative approach. Phys A. (2005) 350:199-206. doi: 10.1016/j.physa.2004.11.041

14. Ebaid A. Analysis of projectile motion in view of the fractional calculus. Appl Math Model. (2011) 35:1231-9. doi: 10.1016/j.apm.2010.08.010

15. Garcia JJR, Calderon MG, Ortiz JM, Baleanu D. Motion of a particle in a resisting medium using fractional calculus approach. Proc Roman Acad Ser A. (2013) 14:42-7.

16. Ebaid A, Masaedeh B, El-Zahar E. A new fractional model for the falling body problem. Chin Phys Lett. (2017) 34:020201. doi: 10.1088/0256-307X/34/2/020201

17. Alharbi FM, Baleanu D, Ebaid A. Physical properties of the projectile motion using the conformable derivative. Chin J Phys. (2019) 58:18-28. doi: 10.1016/j.cjph.2018.12.010
18. Kac VG, Cheung P. Quantum Calculus. New York, NY: Springer-Verlag (2002).

Conflict of Interest: The authors declare that the research was conducted in the absence of any commercial or financial relationships that could be construed as a potential conflict of interest.

Copyright (c) 2020 Alanazi, Ebaid, Alhawiti and Muhiuddin. This is an open-access article distributed under the terms of the Creative Commons Attribution License (CC $B Y)$. The use, distribution or reproduction in other forums is permitted, provided the original author(s) and the copyright owner(s) are credited and that the original publication in this journal is cited, in accordance with accepted academic practice. No use, distribution or reproduction is permitted which does not comply with these terms. 